\title{
The Efficacy of Epidural Steroid In Management of Chronic Low Back Ache
}

\author{
${ }^{* 1} J a c o b$ Ipe, ${ }^{2}$ VivianD’Almeida, JacobEapen \\ Father Muller Medical College,Mangalore \\ *Corresponding author: Jacob Eapen*
}

\section{Introduction}

It is a well recognized fact that back pain in a disc disease is due to chemical inflammation in addition to compression of neural elements ${ }^{[1]}$.Surgery aims at decompressing the pressure on neural elements and conservative treatment aims to combat the chemical radiculitis. Since the advent of epidural analgesia by Corning in 1885 epidural medications have been used in the treatment of this condition for over 100 years. However, controversy still persists regarding their effectiveness in reducing the pain and improving function. This study aims at analyzing the effectiveness of epidural steroid in a diagnosed and confirmed case of lumbar disc herniation.

\section{Aims And Objectives:}

To compare and analyse the effectiveness of epidural steroid and to study the role of lifestyle (such as smokers and obese individuals) in the outcome of efficacy of steroids via epidural route and to compare their results

\section{Materials And Methods}

A prospective study of one hundred patients with lumbar disc herniations in the age group of 25-70 years were selected. Period of study was one year from August 2015 to July 2016.Patients who were presented to the department of Orthopaedics at Father Muller Medical College hospital,Mangalore were drawn into the study.

The Inclusion Criteria for selection of patients were

1) Patients above 18 years of age treated by only nonoperative methods.

2) Symptomatic patients with disc herniations with positive MRI findings.

3) Failure after a minimum of 8 weeks of conservative treatment

4) No history of lumbar surgery

Patients excluded from the study were:

1) Patients below 18 years.

2) Patients with progressive neurological deficits.

3) Patients with associated listhesis,instability of the spine.

4) Patients with caudaequina lesions.

5) Patients with history of previous spine surgery

6) Patients associated with infection or discitis

7) Any condition that comes in the way of early mobilization of the patient.

All patients suffering from back pain with or without radiation to lower limbs were subjected to detailed clinical history and examination. X-ray and MRI of lumbosacral spine were done in every case and those having a prolapsed lumbar disc as the cause of back pain were selected. All selected patients were informed about the study.

Patients planned for epidural steroid injections were briefed about the pain management and goals and a written consent taken. Platelet inhibitors were stopped a week prior to surgery, systemic infections were ruled out before injecting epidural steroid. Epidural steroid injection was performed by the anaesthetist on call in the operation theatre with patient positioned in lateral position. Injection containing Triamcinolone 40mg mixed with 3 to $4 \mathrm{ml}$ of $2 \%$ lignocaine was injected to the epidural space,without fluoroscopic guidance,by interlaminar approach.All these injections were performed at one level cephalad to the disc herniation. 


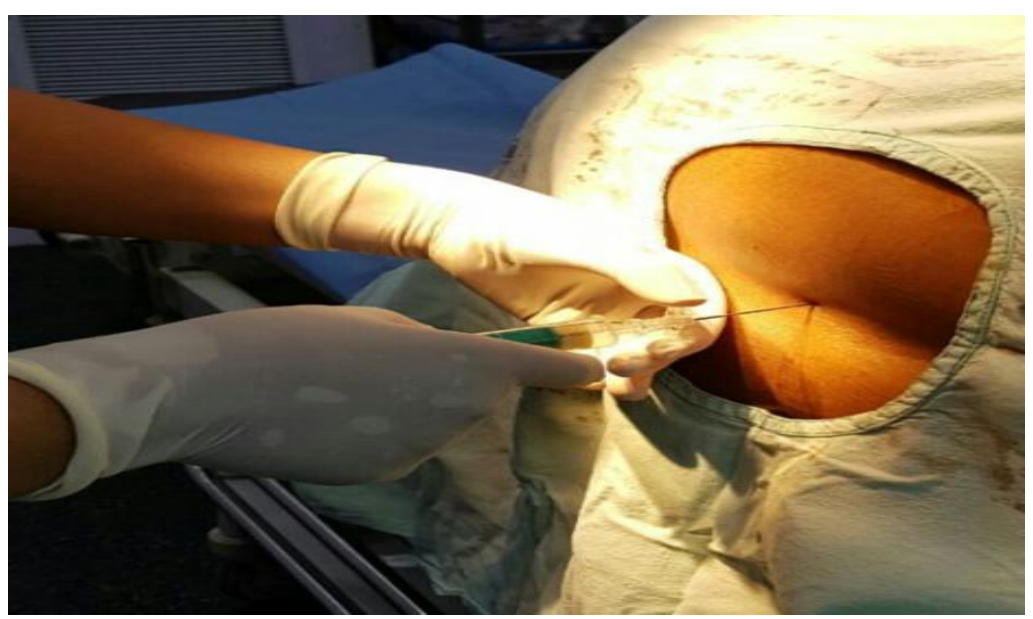

Figure No.1: Administering epidural steroid

All patients were prospectively assessed with clinical examination and questionnaires which included a visual analogue scale of 0 to 10 for assessment of current back and radicular pain and an Oswestry disability index was used to quantitate the level of function. The questionnaire and clinical examination was completed at presentation and at every follow up clinical visit .Follow up was carried out at one, six and twelve months interval after treatment.The collected data was analysed by frequency percentage and chi square test using SPSS version 20 software to evaluate the results.

\section{Results}

Our results showed that in both genders, epidural steroid injection yielded better results in female patients compared to the male patients as their Oswestry disability index(ODI) got better at 6 months follow up.Patients who had a sedentary life style recovered better with epidural steroid injection as compared to the heavy labour group of patientsAmongst smokers, the level of disability was noted to be higher when a smoker had lumbar disc herniation (worse initial ODI scores) as compared to non smokers. Also, there was a significant delay in the recovery as compared with the non smoker group $36 \%$ of patients only had lumbar disc bulge, $8 \%$ had extrusion and $56 \%$ had protrusion in our study. We found that irrespective of disc bulge, protrusion or extrusion the epidural steroid injection group showed significant improvement in symptoms. Hence, the magnitude of disc herniation is not directly proportional to the outcome. We did not notice any complications in patients treated with epidural steroid injection viz.,headache,facial flushing, back pain,nerve deficits and injury, injection,epidural haematoma,etc.

\section{Discussion}

Sciatica due to lumbar intervertebral disc herniation is one of the most common cause of radicular pain in an adult working population. ${ }^{[2,3]}$ Many patients have a tendency to avoid surgery considering its associated risk factors and cost. Solberg et al.,in their study, reported a $4 \%$ risk of worsening of symptoms after a lumbar discectomy ${ }^{[4]}$ Lumbar epidural steroid injection is a reasonable non surgical option available in such situation. ${ }^{[5,6,7]}$

Thirty six percent of patients had either protrusion or extrusion of the lumbar disc. Irrespective of the magnitude of disc herniation, epidural steroid injection showed significant improvement in symptoms. ${ }^{[8]}$ Beyond doubt steroid definitely helps control the chemical inflammation causing nerve root irritation which is believed to play a critical role in the genesis of radicular pain with and without the presence of mechanical compression of the nerve roots or cord. ${ }^{[9]}$

We observed the fact that after a dose of epidural steroid injections the ODI got better and better with time. Though patient had a good relief from the radicular pain immediately following the injection we noticed that the sense of satisfaction of well being was only after few months. The quick relief can probably be attributed to the local anaesthetic that is injected along with the steroid. Swerdlow.M.et al in his study of intradiscal medications in the relief of lumbosciatic syndrome states that methylprednisolone injected in the epidural space can remain inside forupto 2 weeks ${ }^{[10]}$. Probably this could explain the delayed action of epidural steroid. Abdi S, in his systematic review, found strong evidence for short term pain relief and moderate evidence for long term pain relief by use of epidural steroids ${ }^{[11]}$ Similar results were reported by many other studies ${ }^{[12,13]}$ Manchikanti et al., do not report any major adverse event in their study and assures the safety of the injection techniques ${ }^{[14]}$ 
$44 \%$ of our patients were into heavy physical activity and $56 \%$ were having sedentary lifestyle. We observed that outcomes were poor irrespective of treatment given in patients who had sedentary lifestyle. This probably stresses the need for a regular daily muscular stretching and strengthening exercise program for long term favourable outcome and well being. We did not notice any significant difference in the outcome of patients with a longer duration of symptoms when compared with those who had shorter duration of symptoms.Holm $\mathrm{S}$ et $\mathrm{al}^{[15]}$ in his study on the effects of cigarette smoking on nutrition of the intervertebral disc states that smoking causes impairment of disc nutrition. Furthermore,Iwahashi et al ${ }^{[16]}$ in his study of mechanism of disc degeneration states that smokers have decreased oxygen levels leading to hyalinization and necrosis of the nucleus pulposus.True to the above two studies we found that non-smokers had better outcome than smokers. This is consistent with other studies. Also,we noted that smokers had worse Oswestry disability scores initially itself suggesting that smokers had more disability with respect to disc herniation. For those with very bothersome symptoms,epidural steroid would hasten the recovery and may result in better outcomes ${ }^{[17]}$.

\section{Conclusion}

The management of low back pain and radicular pain due to a prolapsed lumbar intervertebral disk by injecting steroid in epidural space is satisfactory in the current study. It is not a new technique but deserves a wider use and scientific assessment. Epidural steroid injection probably accelerates pain relief in patients who eventually have natural resolution of radicular pain in a gradual delayed fashion and allow faster return to function during the natural history of sciatica. The results obtained suggest a more rational use of the epidural steroid which should in turn give better results. It can be considered to be a good supportive and symptomatic treatment option and can avoid countless days of disability and unnecessary hospital stay. Patient education with respect to injury avoidance and home therapeutic exercise program is definitely an effective means in treating the symptoms of lumbar disc diseases. Cessation of smoking should be an integral part of the treatment of patients with lumbar disc diseases.

\section{References}

[1]. Putti V:New conceptions in the pathogenesis of classic pain.1927.Lancet 9:53-60

[2]. Walker BF.The prevalence of low back pain: a systematic review of the literature from 1966 to 1998.J Spinal Disord.2000;13:205-17.

[3]. Damian H, Christopher B, Gail W, Lyn M, Peter B. A systematic review of global prevalence of low back pain. Arthritis and Rheumatism. 2012;64:2028-37

[4]. Tore KS,OysteinPN,KristinS,DagH,Tor I. The risk of "getting worse" after lumbar microdiscectomy.Eur Spine J. 2005;14(1):49-54

[5]. Corning JL. Spinal anaesthesia and local medication of the spinal cord.New York Medical Journal1885;31:483-85

[6]. Glen R Buttermann. Treatment of lumbar disc herniation. Epidural steroid injection compared with disectomy, JB \& JS, 2004, 86: $670-679$.

[7]. Laxmaiah M, Vijay S, Vidyasagar P, Frank JE, Joshua A. Comparison of the efficacy of Caudal, Interlaminar, and Transforaminal epidural Injections in managing lumbar disc herniation: Is one method superior to the other? Korean JPain. 2015;28:11-21.

[8]. Lievre JA ,Block -Michel H,Attali P(1957):L' Injection transacreeEtudeClinique at Radiologique,BullSoc Med Hosp(Paris) 73:1110-1117

[9]. RozenbergS,DuborgG,KhalifaP,etal:Efficacy of epidural steroids in low back pain and sciatica:A critical appraisal by a French task force of randomnizedtrials. RevRhum 1999, 66:79-85.

[10]. Swerdlow et al :The duration of action of bupivacaine,prilocaine and lignocaine(Br.J.Anaes 42:335-339,1970

[11]. Saal JA, Saal JS, Herzog RH. The natural history of lumbar intervertebral disc extrusions treated nonoperatively.Spine1990;15:683-86

[12]. Chou R, Atlas SJ, Steven MPH, et al. Nonsurgical interventional therapies for

[13]. low-back pain. A review of the evidence for an American Pain Society Clinical Practice guideline.Spine. 2009;34:107893.

[14]. Spaccarelli KC. Lumbar and caudal epidural corticosteroid injections.MayoClin Proc. 1996;71:169-78.

[15]. brahimBurak,Atci,Albayanak,Serdal A:Journal of Neurology Research 2013 December Vol3,Issue 6 p173.

[16]. Holm S et al Nutrition of the intervertebral disc:Solute,transport and metabolism;1981 Vol 8 No.2 101-119 Bone and joint research,London,England

[17]. Iwahashi et al :Mechanism of intervertebral disc degeneration caused by nicotine in rabbits to explicate intervertebral disc disorders caused by smoking;Issue 13-Vol 27 1396-1401 July 2002

[18]. Wang, Jeffrey C; Lin, Eric; Brodke, Darrel S: Epidural Injections for the Treatment of Symptomatic Lumbar Herniated Discs,2002 Journal of Spinal disorders and techniques Aug 2002-Vol 15:Issue 4:pp 269-272.

\footnotetext{
* Jacob Ipe. "The Efficacy of Epidural Steroid In Management of Chronic Low Back Ache." IOSR Journal of Dental and Medical Sciences (IOSR-JDMS) 16.7 (2017): 14-16.
} 